\title{
Eighteen-Year Disease Progression and Survival in CADASIL
}

\author{
Gido Gravesteijn, ${ }^{\text {a }}$ Remco J. Hack, ${ }^{\text {a } A n n a ~ M . ~ v a n ~ O p s t a l, ~}{ }^{\mathrm{b}}$ Bastian J. van Eijsden, ${ }^{\mathrm{a}}$ Huub A.M. Middelkoop, ${ }^{\mathrm{c}, \mathrm{d}}$ \\ Mar D.M. Rodriguez Girondo, ${ }^{\mathrm{e}}$ Annemieke Aartsma-Rus, ${ }^{\mathrm{f}}$ Jeroen van de Grond, ${ }^{\mathrm{b}}$ Julie W. Rutten, ${ }^{\mathrm{a}, \mathrm{f}}$ \\ Saskia A.J. Lesnik Oberstein ${ }^{\mathrm{a}}$

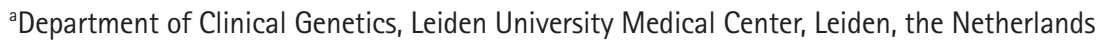 \\ ${ }^{b}$ Department of Radiology, Leiden University Medical Center, Leiden, the Netherlands \\ 'Department of Neurology, Leiden University Medical Center, Leiden, the Netherlands \\ 'Institute of Psychology, Health, Medical and Neuropsychology Unit, Leiden University, Leiden, the Netherlands \\ eDepartment of Biomedical Data Sciences, Leiden University Medical Center, Leiden, the Netherlands \\ fDepartment of Human Genetics, Leiden University Medical Center, Leiden, the Netherlands
}

\section{Dear Sir:}

Cerebral autosomal dominant arteriopathy with subcortical infarcts and leukoencephalopathy (CADASIL), the most prevalent hereditary cerebral small vessel disease, is typically associated with mid-adult onset of strokes and vascular cognitive decline leading to dementia and complete care-dependency. A severe disease course is associated with cysteine-altering variants in epidermal growth-factor repeat (EGFr) domains 1-6 of the NOTCH3 protein, whereas variants located in EGFr domains 7-34 are associated with a milder phenotype. ${ }^{1}$ However, even individuals with identical mutations can have variable disease courses. Hypertension has been shown to contribute, ${ }^{2}$ but there are likely other important modifiers.

To investigate very long-term disease progression and variability, we performed an 18-year follow-up study of the original CADASIL2000 baseline cohort. ${ }^{3} \mathrm{~A}$ strength is that individuals of various ages (22 to 51 years) and disease-stages (presymptomatic to disabled) were included and all had a comprehensive and uniform study protocol at baseline ${ }^{3}$ and 18-year follow-up. Our primary aim was to assess long-term disease outcome and variability, while taking into account mutation position. A secondary aim was to determine whether the main clinical predictors of shorter-term disease outcome, i.e., lacune count and brain parenchymal fraction (BPF) ${ }_{1}^{4}$ also determine long-term disease course.

At 18-year follow-up, 22/41 (54\%) patients were still alive (mean \pm standard deviation age, $57.6 \pm 8.8$ years [range, 40 to 70]). Fifteen surviving individuals consented to participate in the full on-site study protocol, including magnetic resonance imaging and neuropsychological testing. Remarkably, more than half of these individuals (8/15), all at an age when most CADASIL patients are expected to be symptomatic, had remained clinically stable over almost two decades, with no or only very mild disability at follow-up (modified Rankin Scale $[\mathrm{mRS}] \leq 1$ ) and with no significant cognitive deterioration compared to the healthy controls, even on executive function tests (Trail Making Test-B [TMT-B] and Wechsler Adult Intelligence Scale substitution test [WAIS substitution]). This is surprising, as executive function has been described to be the first cognitive domain to be affected, even in pre-stroke patients. However, previous studies did not take the presence of lacunes into account, whereas we found that clinically stable patients showed no difference in rate-of-increase of white matter hyperintensity (WMH) volume compared to individuals who had significant progression on disability and cognitive scales (median, 27.5\%o [range, 15.1 to 36.2 ] vs. $23.9 \%$ [range, 15.2 to 38.9], $P=0.95$ ), but they did have significantly less increase of lacunes (median, 2 lacunes [range, 0 to 9] vs. 6.5 lacunes [range, 1 to 12 ], $P=0.07$ ), corroborating the hypothesis that cognitive (executive) function and $m R S$ remain largely intact in the pre-lacune stage, irrespective of WMH burden. Unexpectedly, stable patients did not harbor EGFr 7-34 mutations (6/8 had a mutation in one of EGFr domains 1-6), nor did they have

Copyright () 2021 Korean Stroke Society

This is an Open Access article distributed under the terms of the Creative Commons Attribution Non-Commercial License (http://creativecommons.org/licenses/by-nc/4.0/) which permits unrestricted non-commercial use, distribution, and reproduction in any medium, provided the original work is properly cited. 
less cardiovascular risk factors than progressors. As is the case for any slowly progressive disorder, age is a strong predictor of disease progression. However, some patients with the same age at baseline had extreme variation in long-term disease progression, with some remaining stroke- and lacune-free well into the sixth decade, while others were already deceased for some years (Figure 1).

The 19 patients who did not survive to 18-year follow-up had died at an average age of $62.4 \pm 7.1$ years (range, 50 to 75). Univariate analysis of baseline variables showed that the following were associated with mortality: age $(P=0.005)$, presence of stroke $(P=0.02)$, lacune count $(P<0.001)$, and disability (mRS) $(P<0.001)$. Patients with no lacunes or disability at baseline had high survival rates, namely more than $80 \%$ over 18 years, whereas patients with moderate-severe disability (mRS 3-5) at baseline, who all had lacunes, had estimated survival rates of $18 \%$ and a median survival time of 7.7 years. Only lacune count (hazard ratio [HR], 1.074 per additional lacune; 95\% confidence interval $[\mathrm{Cl}], 1.03$ to $1.12 ; P=0.001)$ and age $(\mathrm{HR}$, 1.11 per year; $95 \% \mathrm{Cl}, 1.02$ to $1.20 ; P=0.02$ ) were significantly associated with mortality in a forward multivariate analysis including age, sex, lacune count, WMH volume, and BPF.

Of the 30/41 patients who had a history of at least one stroke, mean age at first stroke was 49.9 years $(95 \% \mathrm{Cl}, 46.1$ to 53.7; range, 31 to 67). As was previously reported, ${ }_{1}^{1}$ age at first stroke was younger in individuals with an EGFr 1-6 variant compared to those with an EGFr 7-34 variant, but this was not statistically significant in this cohort (48.6 years [95\% Cl, 44.6
Individual 1
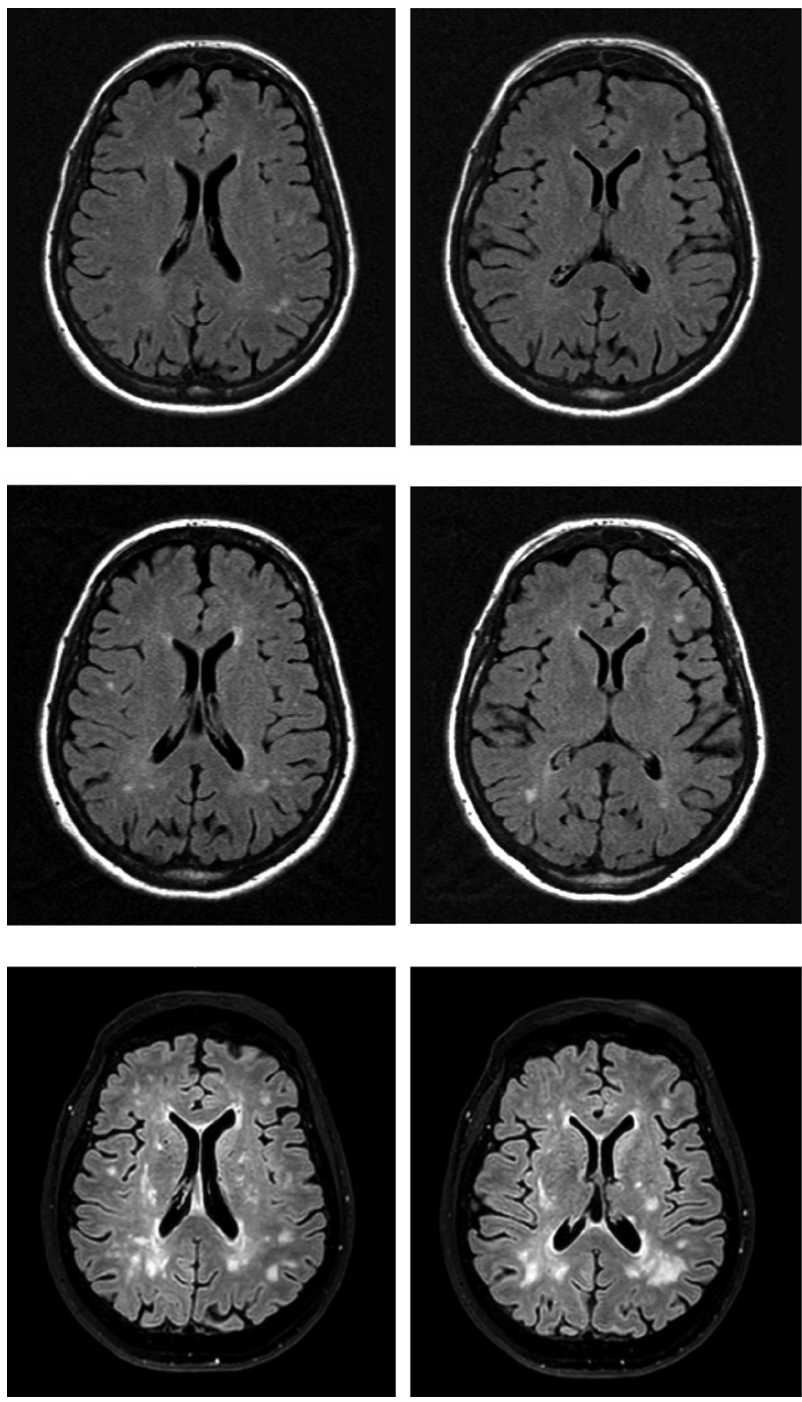

Individual 2
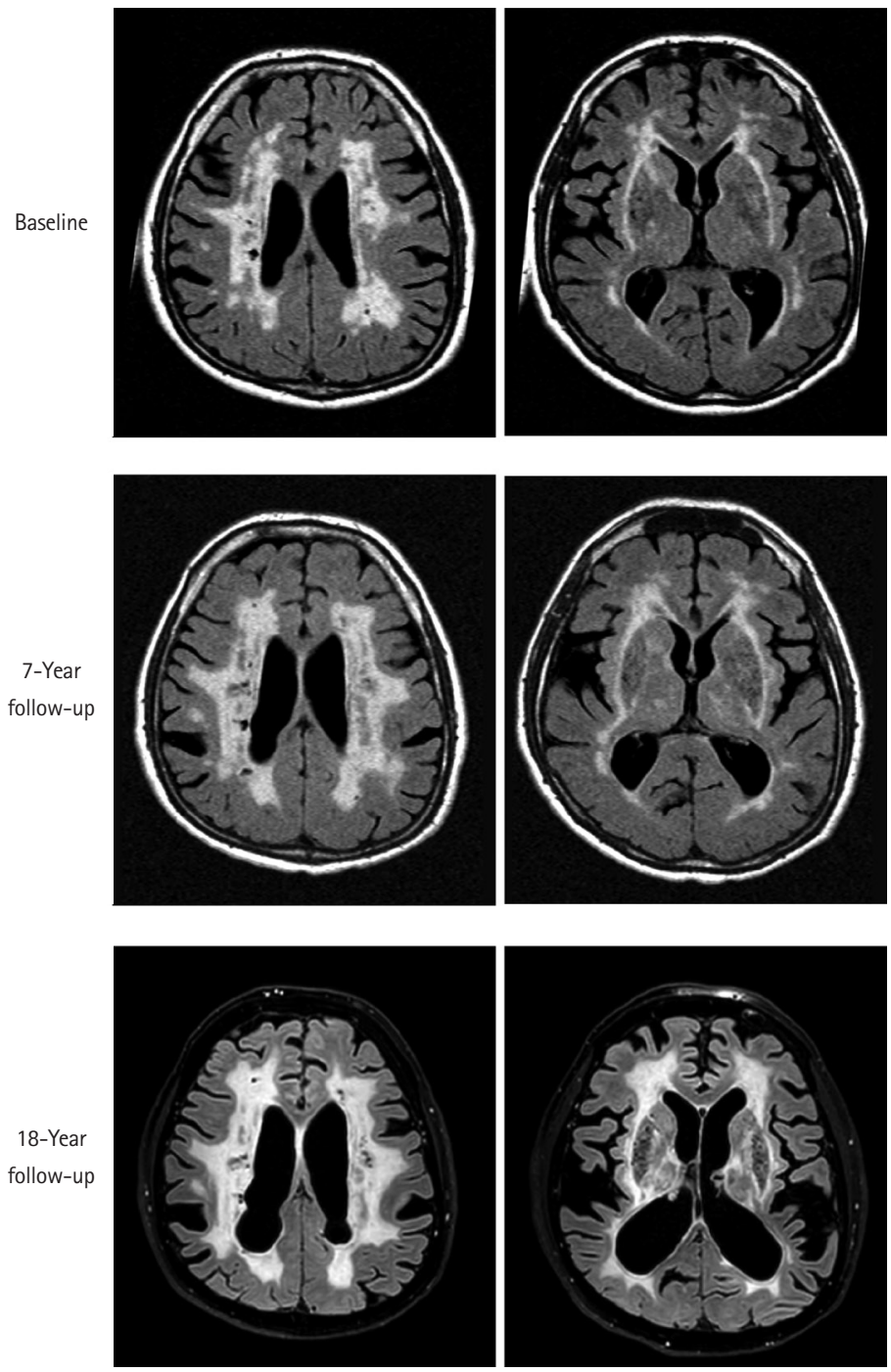

B

Figure 1. Magnetic resonance fluid-attenuated inversion recovery (FLAIR) images of two individuals of same age with a NOTCH3 variant in in epidermal growth-factor repeat 7-34: (A) clinically stable versus (B) progressive 18-year course. 
to 52.7$]$ vs. 53.0 years [ $95 \% \mathrm{Cl}, 43.7$ to 62.3$], P=0.31$ ).

In conclusion, although the numbers are inherently small in a long-term single-center follow-up of a severe disease, these data show that in a sample of CADASIL patients of the first hour, two decade disease progression is remarkably variable and this variability is not fully explained by mutation position or cardiovascular risk factors. The patients remaining long-term clinically stable suggest that protective factors may play an important role, even in those individuals with severe mutations. Identifying these protective factors may be a first step in developing future preventive treatment for individuals with $\mathrm{NOTCH} 3$ cysteine altering variants, as well as further refining disease prediction models.

\section{References}

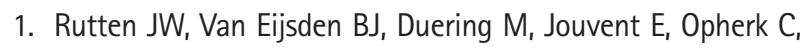
Pantoni $L$, et al. The effect of NOTCH3 pathogenic variant position on CADASIL disease severity: NOTCH3 EGFr 1-6 pathogenic variant are associated with a more severe phenotype and lower survival compared with EGFr 7-34 pathogenic variant. Genet Med 2019;21:676-682.

2. Adib-Samii P, Brice G, Martin RJ, Markus HS. Clinical spectrum of CADASIL and the effect of cardiovascular risk factors on phenotype: study in 200 consecutively recruited individuals. Stroke 2010;41:630-634.

3. van den Boom R, Lesnik Oberstein SA, Ferrari MD, Haan J, van Buchem MA. Cerebral autosomal dominant arteriopathy with subcortical infarcts and leukoencephalopathy: MR imaging findings at different ages. 3rd-6th decades. Radiology 2003;229:683-690.

4. Jouvent E, Duchesnay E, Hadj-Selem F, De Guio F, Mangin JF, Hervé $D$, et al. Prediction of 3-year clinical course in CADASIL. Neurology 2016;87:1787-1795.
Correspondence: Saskia A.J. Lesnik Oberstein

Department of Clinical Genetics, Leiden University Medical Center, P0 Box 9600, 2300 RC Leiden, the Netherlands

Tel: $+31-71-5268033$

Fax: +31-71-5266749

E-mail: lesnik@lumc.nl

https://orcid.org/0000-0002-1268-8995

Co-correspondence: Julie W. Rutten

Department of Clinical Genetics, Leiden University Medical Center, P0 Box 9600, 2300 RC Leiden, the Netherlands

Tel: $+31-71-5268033$

Fax: +31-71-5266749

E-mail:j.w.rutten@lumc.nl

https://orcid.org/0000-0003-3250-7263

Received: October 9, 2020

Revised: November 9, 2020

Accepted: November 18, 2020

This work was funded by the Netherlands Brain Foundation (HA2016-02-03; BG2015-2; Gido Gravesteijn and Julie W. Rutten). The authors thank all CADASIL patients and their family members who participated in the study. We acknowledge Merve Atli, Lesse Tieman, Hella Janssen, Annick den Hartog, and Yvette Knaap for conducting the neuropsychological assessments.

NOTCH3 antisense therapies have been patented by the Leiden University Medical Center. As co-inventors on this patent Annemieke Aartsma-Rus and Saskia A.J. Lesnik Oberstein are entitled to a share of potential royalties. Remco J. Hack was funded by Dutch National Institutes of Health (ZonMW, reference 91717325). Annemieke Aartsma-Rus reports being employed by Leiden University Medical Center (LUMC) which has patents on exon skipping technology, some of which has been licensed to BioMarin and subsequently sublicensed to Sarepta. As co-inventor of some of these patents Annemieke Aartsma-Rus is entitled to a share of royalties. Annemieke Aartsma-Rus further discloses being ad hoc consultant for PTC Therapeutics, Sarepta Therapeutics, CRISPR Therapeutics, Summit PLC, Alpha Anomeric, BioMarin Pharmaceuticals Inc., Eisai, Astra Zeneca, Santhera, Audentes, Global Guidepoint and GLG consultancy, Grunenthal, Wave and BioClinica, having been a member of the Duchenne Network Steering Committee (BioMarin) and being a member of the scientific advisory boards of ProQR, Hybridize Therapeutics, Silence Therapeutics, Sarepta Therapeutics, and Philae Pharmaceuticals. Remuneration for these activities is paid to LUMC. LUMC also received speaker honoraria from PTC Therapeutics and BioMarin Pharmaceuticals and funding for contract research from Italpharmaco and Alpha Anomeric. 